\title{
Can regular moderate exercise lead to changes in miRNA-146a and its adapter proteins in the kidney of streptozotocin-induced diabetic male rats?
}

\author{
${ }^{1}$ Oghbaei H, ${ }^{1}$ Ahmadi Asl N, ${ }^{2}$ Sheikhzadeh F \\ ${ }^{1}$ Drug Applied Research Center, Tabriz University of Medical Sciences, Tabriz, Iran \\ ${ }^{2}$ Department of Animal Biology, Faculty of Natural Sciences, University of Tabriz, Tabriz, Iran \\ E-mail:n.ahmadiasl@gmail.com
}

Objective. The aim of this study was to assess whether microRNA-146a and its adapter proteins TNF receptor associated factor6 (TRAF6) and interleukin-1 receptor-associated kinase-1 (IRAK1) may be changed in the kidney of streptozotocin-induced diabetic rats, following regular moderate exercise.

Methods. Forty adult male Wistar rats were allocated randomly into four groups $(\mathrm{n}=10)$, including sedentary control (SC), sedentary diabetic (SD), healthy sixty-day exercise (H60E), and diabetic sixty-day exercise (D60E) groups. Diabetes was induced by an intraperitoneal injection of $60 \mathrm{mg} / \mathrm{kg}$ streptozotocin. After $48 \mathrm{~h}$, blood glucose levels $>250 \mathrm{mg} / \mathrm{dl}$ was included to diabetic rats. After 2 days of diabetes induction, the exercise protocol began. Animals were exposed to 5 days of consecutive treadmill exercise for $60 \mathrm{~min} /$ day with the $22 \mathrm{~m} / \mathrm{min}$ speed for 60 days. The kidneys of the rats were removed and microRNA was extracted from them using the miRCURYTM RNA isolation kit.

Results. In diabetic rats, statistical analysis revealed a significant decrease in miR-146a expression, non-significant decrease in IRAK1 mRNA expression, and non-significant increase in TRAF6 and NF-kB mRNA expression compared to the SC group. Exercise led to a non-significant increase in the expression of miR-146a and NF-kB mRNA in the kidneys of the diabetic group as compared to the SD group, significant increase in TRAF6 and IRAK1 mRNA expression compared to the H60E group, and significant increase in TRAF6 mRNA expression compared to the SD group.

Conclusion. The present data indicate that exercise might be able to help in the prevention in the diabetic nephropathy development.

Key words: diabetes, exercise, kidney, miRNA-146a

Diabetes mellitus is a metabolic disorder characterized by a high blood glucose (Umpierrez and Korytkowski 2016). Hyperglycemia leads to the activation of several cellular pathways, increased polyol pathway flux, increased production of advanced glycation end products, MAPK signaling pathway, activation of protein kinase $\mathrm{C}$, and an ultimate increase in the activation of the nuclear factor kappa B (NF-kB) (Safi et al. 2014). NF-kB is a pro-inflammatory transcription factor which has a critical role in the pathogenesis of diabetic complications, including nephropathy (LuisRodriguez et al. 2012) and can also lead to a change in the expression of some miRNAs, such as miR-146a, miR-132 and miR-155 (Asirvatham et al. 2009). MiRNAs, a group of small non-coding RNAs, composed of approximately 22 nucleotides, are the main regula- 
tors of gene expression by binding to the 3'UTR section of mRNAs and inhibiting them or destructing their cellular translation (Pasquinelli 2012). They play a crucial role in the etiology and pathogenesis of many diseases including diabetes mellitus (Chen et al. 2014; Moura et al. 2014). Recent findings have revealed very important functions for special miRNAs in several biological and cellular processes, including differentiation, proliferation, and development of genes related, and the regulator of immune responses (Croce and Calin 2005; Chen 2005; Baxter et al. 2012). MiR-146a, a known anti-inflammatory microRNA, has been found to be functionally important in the diabetic nephropathy (Bhatt et al. 2016). MiR-146a is a NF-kB dependent gene with two known targets, TRAF6, and IRAK1. Both TRAF6 and IRAK1 targets are down-regulated by miRNA-146a (Saba et al. 2014). Balasubramanyam et al. (2011) have show that devastated miR-146a expression links to insulin resistance and subclinical inflammation in diabetic patients. Feng et al. (2011) have demonstrated that miR-146a takes part in the regulating of the extracellular matrix protein generation in the retina, heart, and kidney of the diabetic rats. Alipour et al. (2013) have also revealed that the expression of miRNA-146 is altered in the diabetic kidney. Nephropathy is a perilous complication of typel and type2 diabetes since it can ultimately lead to end-stage renal failure (Remuzzi et al. 2006). For decades, exercise has been considered as a cornerstone of diabetes management along with the diet planning and medication (Horton 1988; Hayes and Kriska 2008; Praet and van Loon 2008; Dogan et al. 2010). Even though, some studies have suggested that exercise accelerates diabetic nephropathy progression (Mogensen et al. 1983; Matsuoka et al. 1991). Several studies in diabetic animals have indicated that exercise may improve metabolic control, decreases proteinuria, and has a beneficial effect on the renal function (Ward et al. 1994; Chiasera et al. 2000; dos Santos Silva et al. 2012). It is believed that these differences can be due to the variety in the duration and intensity of exercise (Virvidakis et al. 1986; Poortmans et al. 1996). Recent studies have demonstrated that chronic exercise has beneficial effects that reduce the early progression of diabetic nephropathy (Tufescu et al. 2008; Ito et al. 2015). In recent years, research interest has been increased in the field of aspects related to the body's response to exercise, in which miRNAs appear to play a key role. Some new studies have recently revealed that exercise can modulate the expression of some miRNAs in certain tissues including the skeletal muscle (McCarthy and Esser 2007), heart (Ma et al. 2013), and neutro- phils (Radom-Aizik et al. 2010). The involvement of miR-146a in the pathogenesis of diabetic nephropathy has been previously reported (Alipour et al. 2013; Huang et al. 2014) and showed that it can also affect the expression of many miRNAs (Banzet et al. 2013; Russell et al. 2013; Zacharewicz et al. 2013). Therefore, it is possible that exercise may cause an adjustment in miR-146a expression changes in the diabetic kidney and finally prevent diabetic nephropathy. To assess this hypothesis, we surveyed the expression of miR-146a, TRAF6, IRAK1, and NF-kB in the kidney of diabetic rats following regular moderate exercise.

\section{Materials and Methods}

Subjects. Forty adult-male Wistar rats weighing between $200 \pm 50 \mathrm{~g}$ were obtained from the Razi Institute, Tehran, Iran. The animals were maintained at room temperature $\left(22-25^{\circ} \mathrm{C}\right)$ with $12: 12 \mathrm{~h}$ light-dark cycles and, free access to food and water, and resettled as three animals to a cage. The study protocol was designed in accordance with NIH guidelines and the guidelines of the Ethics committee for the use of animals in research at Tabriz University of Medical Sciences. (No: 91/2-3/3). The animals were allocated randomly into four groups of 10/group, including sedentary control (SC), healthy with 60-day exercise (H60E), sedentary diabetic (SD), and diabetic with 60 -day exercise (D60E) groups. They received an intraperitoneal (i.p.) injection of streptozotocin (STZ) $60 \mathrm{mg} / \mathrm{kg}$ BW dissolved in $0.1 \mathrm{~mol} / \mathrm{l}$ citrate buffer (pH 4.5) after fasting for $12 \mathrm{~h}$ (Ozkaya et al. 2007). Control groups received an i.p. dose of the same volume of citrate buffer. Two days later, blood was collected from the tail vein and blood sugar levels were measured using glucometer (Arkray, Kyoto, Japan). Diabetes mellitus was defined as fasting blood glucose $>250 \mathrm{mg} / \mathrm{dl}$. Animals failing this criterion were excluded from the experiment (Ozkaya et al. 2007). There was no mortality due to good maintenance.

Exercise protocol. Before starting the formal 60 day exercises protocol, animals were familiarized to treadmill running (5-20 min/day) for 5 sequential days. After this period of habituation, the exercised animals performed 5 days of continuous treadmill exercise $(60 \mathrm{~min} /$ day) with the speed of $22 \mathrm{~m} / \mathrm{min}$ during the light phase (Taylor et al. 2003). To warm up the rats, at the beginning of the 60 -min exercise, treadmill speed was adjusted at $5 \mathrm{~m} / \mathrm{min}$ and slowly increased to $22 \mathrm{~m} / \mathrm{min}$. To cool down at the end of the 60 -min exercise, the speed was reduced to $5 \mathrm{~m} / \mathrm{min}$. Moderate electrical shock was used for motivating the animals to run. Control groups were placed on a 
turned-off treadmill for $60 \mathrm{~min} /$ day for 5 days a week. Ultimately, exercised animals were studied $24 \mathrm{~h}$ after their last exercise session.

Measurement of blood glucose level. Blood sugar concentrations of diabetic rats were measured in the blood collected from the tail vein in three periods of morning, at the beginning, middle, and end of each experimental period. The body weight of all rats was also measured.

Sample collection. At the end of the experimental period, all animals were anesthetized by the i.p. administration of $100 \mathrm{mg} / \mathrm{kg}$ ketamine and $5 \mathrm{mg} / \mathrm{kg}$ xylazine at 9:00 a.m. (Atalay et al. 2004). The animals' kidneys were immediately removed, washed in cold $9 \%$ normal saline, and frozen in liquid nitrogen.

Total RNA extraction and real-time PCR. Total RNA (including mRNA and microRNA) was extracted from the kidney tissues using a miRCURYTM RNA isolation kit (Exiqon, Vedbaek, Denmark) according to the manufacturer's protocols (Biyashev et al. 2012; Lasser et al. 2012).The procedure was carried out based on the spin column using a dedicated resin as the separation matrix for RNA from other cell ingredients. RNA content and purity were evaluated with Nanodrop1000 spectrophotometer (Thermo Scientific, Wilmington, USA). The expression profile of miR-146a was performed on total RNA excerpts using worldwide cDNA synthesis kit. Briefly, total RNA comprising microRNA was polyadenylated and cDNA was made using a poly $(\mathrm{T})$ primer with a 30 degenerate anchor and a 50 universal tag (Exiqon, Vedbaek, Denmark). Revert Aid First-Strand cDNA Synthesis Kit (Fermentas GmBH, Leon-Rot, Germany) was utilized with the help of random hex- amer primers and MMLV reverse transcriptase (as a comprehensive system for the efficient synthesis of first-strand cDNA from mRNA or total RNA templates) for characterizing IRAK1, TRAF6, and NF-kB mRNA expression levels. Each cDNA was employed as a pattern for separate assays for microRNA and mRNA quantitative real-time PCR using SYBR Green master mix (Exiqon, Vedbaek, Denmark). Locked nucleic acid forward and reverse primer sets (Exiqon, Vedbaek, Denmark) for microRNA and messenger RNA are mentioned in Table 1. Real-time PCR reactions were performed on a Bio-Rad iQ5 detection system (Bio-Rad, Richmond, CA, USA). A number of PCR products were normalized with housekeeping beta-glucuronidase gene for mRNA samples and rnomiR-191 for miR-146a (Peltier and Latham 2008). The 2-(DDCt) method was utilized to determine relativequantitative levels of individual mRNAs and miR146a. The results are expressed as the fold-difference to relevant controls.

Statistical analysis. Data analysis was performed using the analysis of variance (ANOVA) and the results expressed as mean \pm SD. The post hoc LSD was carried out to determine which condition differed significantly from the others. A level of $p<0.05$ was considered statistically significant.

\section{Results}

Blood glucose and body weight level were evaluated in all groups and the results are presented in Table 2. As we expected, significant an increase was observed in the blood glucose of the SD group compared to the $\mathrm{SC}$ group $(\mathrm{p}<0.05)$ and significant reduction was seen

Table 1

Primer set list for mRNAs and miRNAs

\begin{tabular}{|c|c|c|c|}
\hline Gene name & & Accession number & Primer sequence $^{\mathrm{a}}$ \\
\hline TRAF6 & & NM_001107754.2 & $\begin{array}{l}\text { Sence: 5-CAGTCCCCTGCACATT-3 } \\
\text { Antisence: 3-GAGGAGGCATCGCAT-5 }\end{array}$ \\
\hline IRAK1 & & NM_001127555.1 & $\begin{array}{l}\text { Sence: 5-GCTGTGGACACCGAT-3 } \\
\text { Antisence: 3-GCTACACCCATCCACA-5 }\end{array}$ \\
\hline $\mathrm{NF} \kappa \mathrm{B}$ & & XM_343246.4 & $\begin{array}{l}\text { Sence: 5-AATTGCCCCGGCAT-3 } \\
\text { Antisence: } 3 \text {-TCCCGTAACCGCGTA-5 }\end{array}$ \\
\hline Beta Gusb & & NM_017015.2 & $\begin{array}{l}\text { Sence: 5-GGCTCGGGGCAAATT-3 } \\
\text { Antisence: 3-GGGGCAGCACGAT-5 }\end{array}$ \\
\hline Gene name & Accession number & Target sequence $^{\mathrm{b}}$ & Product name \\
\hline mo-miR-146a & MIMAT0000449 & UGAGAACUGAAUUCCAUGGGUU & hsa-miR-146a, LNA PCR primer set, UniRT \\
\hline mo-miR-191 & MIMAT0000440 & CAACGGAAUCCCAAAAGCAGCUG & hsa-miR-191, LNA PCR primer set, UniRT \\
\hline
\end{tabular}

aSequences were derived from NCBI (www.ncbi.nlm.nih.gov);

${ }^{b}$ Sequences were derived from miRBase (www.mirbase.org) 
in the blood glucose of the D60E group in comparison with the SD group $(\mathrm{p}<0.05)$. Also, the final blood glucose level revealed a significant decrease in comparison with the initial blood sugar level in the D60E group $(\mathrm{p}<0.05)$.

The kidney expression of miR-146a is illustrated in Figure 1. The miR-146a expression level decreased significantly in SD kidneys as compared to SC ones (Figure 1) $(p<0.05)$. Exercise in diabetic rats (D60E) led to a non-significant increase in the kidney expression of miR-146a compared to the SD group ( $p>0.05$ ). Exercise in the kidney of the $\mathrm{H} 60 \mathrm{E}$ group reduced the miRNA-146a expression compared to the SC group, although the difference was not significant $(\mathrm{p}>0.05)$.

TRAF6 mRNA expression level revealed a nonsignificant increase in the SD group compared with the SC group ( $p>0.05)$. Moreover, the D60E group revealed a significant increase compared to $S D$ and H60E groups $(\mathrm{p}<0.05)$ (Figure 2).

IRAK1 mRNA expression level was decreased in the SD group compared with the SC group, but this change was not significant ( $\mathrm{p}>0.05)$. A significant increase was seen in IRAK1 expression in the kidneys of the D60E group compared to the H60E group $(\mathrm{p}<0.05)$ (Figure 3).

The NF-kB mRNA expression level indicated a non-significant increase in the SD group compared to the SC group ( $p>0.05$ ). In addition, NF-kB mRNA expression was non-significantly higher in the kidneys of the D60E group than SD and H60E groups ( $>0.05)$ (Figure 4).

\section{Discussion}

Our findings revealed that the expression level of miR-146a significantly decreased in the kidneys of diabetic rats in comparison with the SD group. Our data are in line with data of Feng et al. (2011) and Balasubramanyam et al. (2011) who have shown the declination in miRNA-146a expression in cardiac, retina, peripheral mononuclear, and renal cells of diabetic patients, which led to the development of diabetes

Table 2

General characteristics of STZ-induced diabetic and healthy rats

\begin{tabular}{lcccc}
\hline Parameter & SC & SD & H60E & D60E \\
\hline Initial Blood Glucose $(\mathrm{mg} / \mathrm{dl})$ & $85.3 \pm 4.3 \#$ & $378.8 \pm 39.1^{*} \$$ & $91.3 \pm 5.2 \#$ & $324.8 \pm 17.7^{*} \$$ \\
Final Blood Glucose $(\mathrm{mg} / \mathrm{dl})$ & $88.3 \pm 5.2 \#$ & $455.5 \pm 33.8^{*} \$$ & $81.5 \pm 3.4 \#$ & $175.8 \pm 34.8^{\star} \# \$$ \\
Final Body Weight $(\mathrm{g})$ & $208.1 \pm 8.1 \#$ & $183.0 \pm 10.9^{*} \$$ & $259.1 \pm 9.7 \#$ & $288.8 \pm 23.5 \#$ \\
\hline
\end{tabular}

Data are means \pm SEM. ${ }^{\star} \mathrm{p}<0.05$ compared with SC group; $\# \mathrm{p}<0.05$ compared with SD group; $\$ \mathrm{p}<0.05$ compared with H60E group. Abbreviations: SC - Sedentary Control; SD - Sedentary Diabetic; H60E - Healthy 60 days Exercise; D60E - Diabetic 60 days Exercise

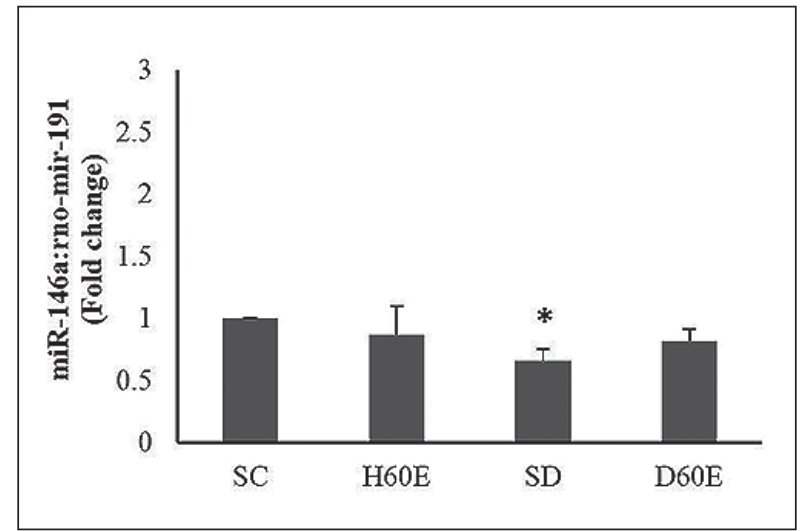

Figure 1. Effect of exercise on miRNA-146a expression in the kidney of diabetic rats. Data are presented as mean \pm S.E. $(\mathrm{n}=6)$, statistical analysis was performed by ANOVA post hoc LSD. ${ }^{*} \mathrm{p}<0.05$ compared with sedentary control group. Abbreviations: SC - sedentary control group; H60E - healthy with 60 -days exercise group; SD - sedentary diabetic group; D60E - diabetes with 60-days exercise group

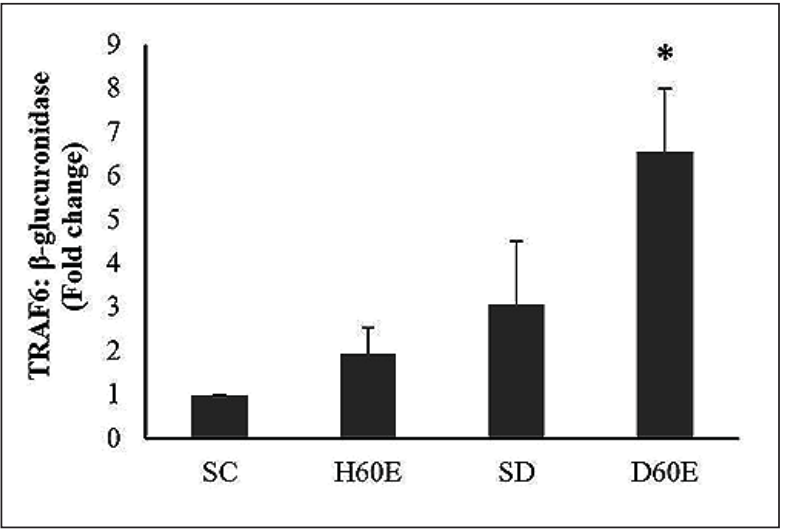

Figure 2. Effect of exercise on TRAF6 expression in the kidney of diabetic rats. Data are presented as mean \pm S.E. $(n=6)$, statistical analysis was performed by ANOVA post hoc LSD. ${ }^{*} p<0.05$ compared with sedentary diabetic group and healthy 60 days exercise group. Abbreviations: SC - sedentary control group; H60E - healthy with 60-days exercise group; SD - sedentary diabetic group; D60E - diabetes with 60-days exercise group 
complications. However, a recent study conducted by Alipour et al. (2013) showed an increased expression of miRNA-146a in diabetic kidneys. Moreover, Rong et al. (2013) have revealed that in patients newly diagnosed with type 2 diabetes mellitus the microRNA146a level was increased in plasma.

Studies demonstrated that through the activation of transcription factor p300 hyperglycemia in diabetes leads to the up-regulation of multiple transcription factors and extracellular matrix proteins, such as fibronectin (Feng et al. 2011). Fibronectin, a glycoprotein of $250 \mathrm{KD}$, is a key component of the extracellular matrix and plays a vital role in the intracellular processes (Pankov and Yamada 2002; Astrof and Hynes 2009). MiR-146a binds to the 3'-UTR of the fibronectin gene and leads to the reduction of the basal fibronectin expression, so that increased fibronectin protein production can be prevented by miR146a mimic transfection (Feng et al. 2011).

Due to the reduced miRNA-146a related to fibronectin mRNA up-regulation in diabetes, fibronectin production and activity increases (Feng et al. 2011). Fibronectin increase results in a decrease in miR146a expression (feedback control) and the decrease in miRNA-146a results in a progress towards diabetic complications (Feng et al. 2011). In addition, we observed an increase in TRAF6 and NF-kB mRNA expression and a decrease in IRAK1 mRNA expression in the SD group; however these changes were not statistically significant. Recent studies have confirmed that miR-146a down-regulated IRAK1 and TRAF6 and then suppressed the NF- $\kappa \mathrm{B}$ promoter-binding

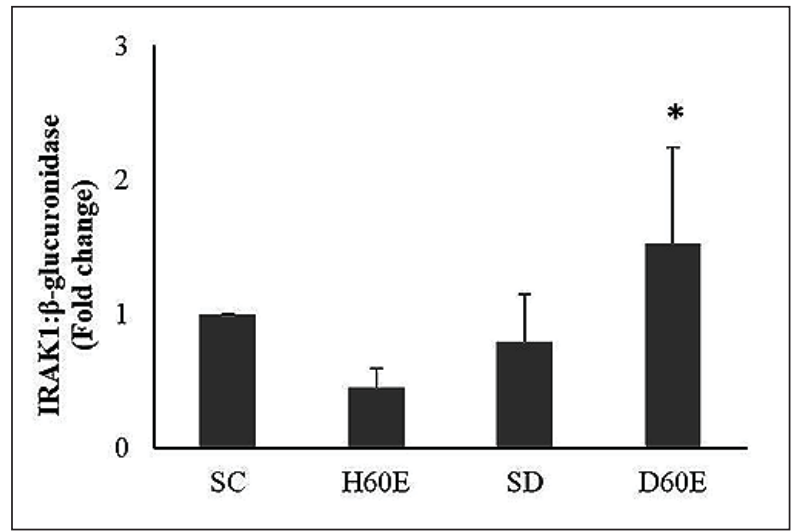

Figure 3. Effect of exercise on IRAK1 expression in the kidney of diabetic rats. Data are presented as mean \pm S.E. $(n=6)$, statistical analysis was performed by ANOVA post hoc LSD. ${ }^{*} \mathrm{p}<0.05$ compared with healthy 60 days exercise group. Abbreviations: SC - sedentary control group; H60E - healthy with 60-days exercise group; SD - sedentary diabetic group; D60E - diabetes with 60-days exercise group activity (Ceribelli et al. 2011; Meisgen et al. 2014). It has been demonstrated that miR-146a induction is dependent on NF-kB and NF- $\kappa B$ activation and induces the transcription of many genes, including IL-6, IL-8, IL-1 $\beta$, TNFa and pri-miR-146a (Taganov et al. 2006; Balasubramanyam et al. 2011). In the present study, as expected, we observed an increase in TRAF6 and NF-kB mRNA expression in the SD group, although these changes were not statistically significant. Nevertheless, we observed no increase in IRAK1 mRNA expression. Several studies have shown that IRAK1 changes were not in line with TRAF6 changes from the miRNA-146a-dependent control loop (Balasubramanyam et al. 2011; Xie et al. 2013). Perhaps the expression of IRAK1 mRNA can be controlled by other factors. Another finding of the present study was a statically non-significant increase in miR-146a level after exercise in the kidneys of diabetic rats, which was accompanied by neither a decreased expression of TRAF6, IRAK1, and NF-kB mRNAs, nor a significant increase in the expression of TRAF6 and IRAK1. Moreover, a non-significant increase was found in NF-kB mRNA. Regardless, the exercise led to an increase in mRNA-146a expression, although it was not significant, but surprisingly TRAF6, IRAK1, and NF-kB mRNA was not decreased. This likely may indicate that the expression of downstream adapter proteins miRNA-146a is also controlled by other factors. Further research is required in order to understand the existence of possible routes, apart from the track of miRNA-146a, for controlling the expression of TRAF6 and IRAK1. Previous studies have shown

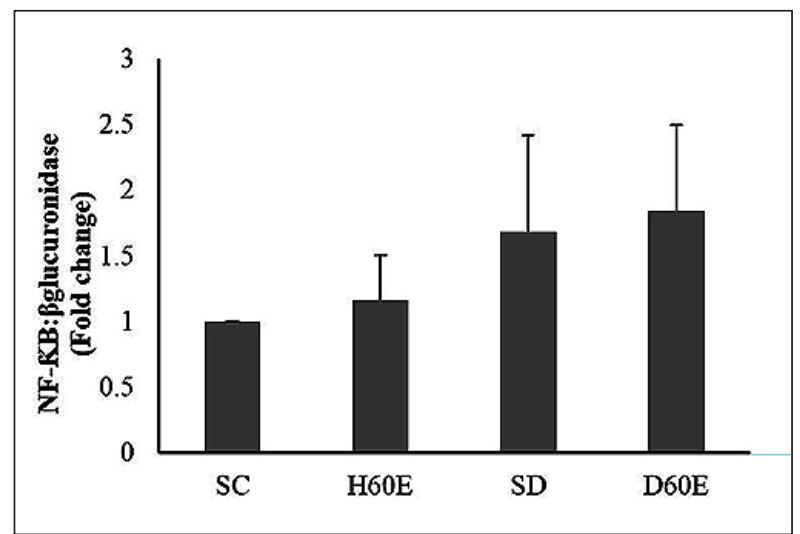

Figure 4. Effect of exercise on NF-kB expression in the kidney of diabetic rats. Data are presented as mean \pm S.E. $(n=6)$, statistical analysis was performed by ANOVA post hoc LSD. Abbreviations: SC - sedentary control group; H60E - healthy with 60-days exercise group; SD - sedentary diabetic group; D60E - diabetes with 60-days exercise group 
that exercise can lead to changes in NF-kB expression (Ji et al. 2004; Fashi et al.2015) and also it has been demonstrated that miR-146a induction is dependent on NF-kB (Taganov et al. 2006; Balasubramanyam et al. 2011). Thus, probably the exercise may lead to changes in miRNA-146a expression through changes in NF-kB expression. Overall, although the variation of miR-146a in the diabetic kidney had previously been reported, we now present the first data on the changes in miR-146a, TRAF6, IRAK1, and NF-kB in the diabetic kidney after regular moderate exercise. These results indicate that although regular moderate exercise increased TRAF6, IRAK1 and NF-kB in the diabetic group, but it could cause an increase in miR-
NA-146a which was reduced by diabetes although that increase was non-significant. These results proved that exercise can prevent diabetic kidneys from the further decrease in miRNA-146a expression and may be helpful to prevent the progression of diabetic nephropathy.

\section{Acknowledgements}

This study was financially supported by Drug Applied Research Center of Tabriz University of Medical Sciences. Our data in this work were derived from the thesis of Ms. Hajar Oghbaei for a Master of Science degree in physiology (thesis serial number: 91/2-3/3).

\section{References}

Alipour MR, Khamaneh AM, Yousefzadeh N, Mohammad-nejad D, Soufi FG. Upregulation of microRNA-146a was not accompanied by downregulation of pro-inflammatory markers in diabetic kidney. Mol Biol Rep 40, 6477-6683, 2013.

Asirvatham AJ, Magner WJ, Tomasi TB. miRNA regulation of cytokine genes. Cytokine 45, 58-69, 2009.

Astrof S, Hynes RO. Fibronectins in vascular morphogenesis. Angiogenesis 12, 165-175, 2009.

Atalay M, Oksala NK, Laaksonen DE, Khanna S, Nakao C, Lappalainen J, Roy S, Hanninen O, Sen CK. Exercise training modulates heat shock protein response in diabetic rats. J Appl Physiol 97, 605-611, 2004.

Balasubramanyam M, Aravind S, Gokulakrishnan K, Prabu P, Sathishkumar C, Ranjani H, Mohan V. Impaired miR146a expression links subclinical inflammation and insulin resistance in Type 2 diabetes. Mol Cell Biochem 351, 197-205, 2011.

Banzet S, Chennaoui M, Girard O, Racinais S, Drogou C, Chalabi H, Koulmann N. Changes in circulating microRNAs levels with exercise modality. J Appl Physiol 115, 1237-1244, 2013.

Baxter D, McInnes IB, Kurowska-Stolarska M. Novel regulatory mechanisms in inflammatory arthritis: a role for microRNA. Immunol Cell Biol 90, 288-292, 2012.

Bhatt K, Lanting LL, Jia Y, Yadav S, Reddy MA, Magilnick N, Boldin M, Natarajan R. Anti-inflammatory role of microRNA-146a in the pathogenesis of diabetic nephropathy. J Am Soc Nephrol 27, 2277-2288, 2016.

Biyashev D, Veliceasa D, Topczewski J, Topczewska JM, Mizgirev I, Vinokour E, Reddi AL, Licht JD, Revskoy SY, Volpert OV. miR-27b controls venous specification and tip cell fate. Blood 119, 2679-2687, 2012.

Ceribelli A, Nahid MA, Satoh M, Chan EK. MicroRNAs in rheumatoid arthritis. FEBS Lett 585, 3667-3674, 2011.

Chiasera JM, Ward-Cook KM, McCune SA, Wardlaw GM. Effect of aerobic training on diabetic nephropathy in a rat model of type 2 diabetes mellitus. Ann Clin Lab Sci 30, 346-353, 2000.

Chen C. MicroRNAs as oncogenes and tumor suppressors. N Engl J Med 353, 1768, 2005.

Chen H, Lan HY, Roukos DH, Cho WC. Application of microRNAs in diabetes mellitus. J Endocrinol 222, R1-R10, 2014.

Croce CM, Calin GA. miRNAs, cancer, and stem cell division. Cell 122, 6-7, 2005.

Dogan A, Ergen N, Kurdak SS. The effects of regular aerobic exercise on renal functions in streptozotocin induced diabetic rats. J Sports Sci Med 9, 294-299, 2010.

dos Santos Silva KA, da Silva Luiz R, Rampaso RR, de Abreu NP, Moreira ED, Mostarda CT, KD Angelis, de Paulo Castro Teixeira V, MC Irigoyen, N Schor. Previous exercise training has a beneficial effect on renal and cardiovascular function in a model of diabetes. PloS One 7, e48826, 2012.

Fashi M, Agha-Alinejad H, Mahabadi HA, Rezaei B, Pakrad BB, Rezaei S. The effects of aerobic exercise on NF- $\kappa B$ and TNF- $\alpha$ in lung tissue of male rat. Novelty in Biomedicine 3, 131-134, 2015.

Feng B, Chen S, McArthur K, Wu Y, Sen S, Ding Q, Feldman RD, Chakrabarti S. miR-146a-mediated extracellular matrix protein production in chronic diabetes complications. Diabetes 60, 2975-2984, 2011.

Hayes C, Kriska A. Role of physical activity in diabetes management and prevention. J Am Diet Assoc 108, S19-S23, 2008. 
Horton E. Exercise and diabetes mellitus. Med Clin North Am 72, 1301-1321, 1988.

Huang Y, Liu Y, Li L, Su B, Yang L, Fan W, Yin Q, Chen L, Cui T, Zhang J, Lu Y, Cheng J, Fu P, Liu F. Involvement of inflammation-related miR-155 and miR-146a in diabetic nephropathy: implications for glomerular endothelial injury. BMC nephrology 15, 142, 2014.

Ito D, Cao P, Kakihana T, Sato E, Suda C, Muroya Y, Ogawa y, HU G, Ishii T, Ito O, Kohzuki M, Kiyomoto H. Chronic running exercise alleviates early progression of nephropathy with upregulation of nitric oxide synthases and suppression of glycation in Zucker diabetic rats. PloS One 10, e0138037, 2015.

Ji LL, Gomez-Cabrera MC, Steinhafel N, Vina J. Acute exercise activates nuclear factor (NF)- $\kappa B$ signaling pathway in rat skeletal muscle. FASEB J 18, 1499-1506, 2004.

Lasser C, Eldh M, Lotvall J. Isolation and characterization of RNA-containing exosomes. JoVE 59, e3037, 2012.

Luis-Rodriguez D, Martinez-Castelao A, Gorriz JL, De-Alvaro F, Navarro-Gonzalez JF. Pathophysiological role and therapeutic implications of inflammation in diabetic nephropathy. World J Diabetes 3, 7-18, 2012.

Ma Z, Qi J, Meng S, Wen B, Zhang J. Swimming exercise training-induced left ventricular hypertrophy involves microRNAs and synergistic regulation of the PI3K/AKT/mTOR signaling pathway. Eur J Appl Physiol 113, 2473-2486, 2013

Matsuoka K, Nakao T, Atsumi Y, Takekoshi H. Exercise regimen for patients with diabetic nephropathy. J Diabet Complications 5, 98-100, 1991.

McCarthy JJ, Esser KA. MicroRNA-1 and microRNA-133a expression are decreased during skeletal muscle hypertrophy. J Appl Physiol 102, 306-313, 2007.

Meisgen F, Landen NX, Wang A, Rethi B, Bouez C, Zuccolo M. MiR-146a negatively regulates TLR2-induced inflammatory responses in keratinocytes. J Invest Dermatol 134, 1931-1940, 2014.

Mogensen C, Christensen C, Vittinghus E. The stages in diabetic renal disease: with emphasis on the stage of incipient diabetic nephropathy. Diabetes 32(Suppl 2), 64-78, 1983.

Moura J, Borsheim E, Carvalho E. The Role of MicroRNAs in Diabetic Complications-Special Emphasis on Wound Healing. Genes 5, 926-956, 2014.

Ozkaya YG, Agar A, Hacioglu G, Yargicoglu P. Exercise improves visual deficits tested by visual evoked potentials in streptozotocin-induced diabetic rats. Tohoku J Exp Med 213, 313-321, 2007.

Pankov R, Yamada KM. Fibronectin at a glance. J Cell Sci 115, 3861-3863, 2002.

Pasquinelli AE. MicroRNAs and their targets: recognition, regulation and an emerging reciprocal relationship. Nat Rev Genet 13, 271-282, 2012.

Peltier HJ, Latham GJ. Normalization of microRNA expression levels in quantitative RT-PCR assays: identification of suitable reference RNA targets in normal and cancerous human solid tissues. RNA 14, 844-852, 2008.

Poortmans JR, Mathieu N, De Plaen P. Influence of running different distances on renal glomerular and tubular impairment in humans. Eur J Appl Physiol Occup Physiol 72, 522-527, 1996.

Praet S, van Loon L. Exercise: the brittle cornerstone of type 2 diabetes treatment. Diabetologia 51, 398-401, 2008.

Radom-Aizik S, Zaldivar F, Oliver S, Galassetti P, Cooper DM. Evidence for microRNA involvement in exerciseassociated neutrophil gene expression changes. J Appl Physiol 109, 252-261, 2010.

Remuzzi G, Macia M, Ruggenenti P. Prevention and treatment of diabetic renal disease in type 2 diabetes: the BENEDICT study. J Am Soc Nephrol 17, S90-S97, 2006.

Rong Y, Bao W, Shan Z, Liu J, Yu X, Xia S, Gao H, Wang X, Yao P, Hu F, Liu L. Increased microRNA-146a levels in plasma of patients with newly diagnosed type 2 diabetes mellitus. PloS One 8, e73272, 2013.

Russell AP, Lamon S, Boon H, Wada S, Guller I, Brown EL, Chibalin AV, Zierath JR, Snow RJ, Stepto N, Wadley GD, Akimoto T. Regulation of miRNAs in human skeletal muscle following acute endurance exercise and shortterm endurance training. J Physiol 591, 4637-4653, 2013.

Saba R, Sorensen DL, Booth SA. MicroRNA-146a: a dominant, negative regulator of the innate immune response. Front Immunol 5, 578, 2014.

Safi SZ, Qvist R, Kumar S, Batumalaie K, Ismail IS. Molecular mechanisms of diabetic retinopathy, general preventive strategies, and novel therapeutic targets. Biomed Res Int 2014, 801269, 2014.

Taganov KD, Boldin MP, Chang KJ, Baltimore D. NF- $\kappa B$-dependent induction of microRNA miR-146, an inhibitor targeted to signaling proteins of innate immune responses. Proc Natl Acad Sci USA 103, 12481-12486, 2006.

Taylor RP, Ciccolo JT, Starnes JW. Effect of exercise training on the ability of the rat heart to tolerate hydrogen peroxide. Cardiovasc Res 58, 575-581, 2003.

Tufescu A, Kanazawa M, Ishida A, Lu H, Sasaki Y, Ootaka T, Sato T, Kohzuki M. Combination of exercise and losartan enhances renoprotective and peripheral effects in spontaneously type 2 diabetes mellitus rats with nephropathy. J Hypertens 26, 312-321, 2008. 
Umpierrez G, Korytkowski M. Diabetic emergencies (mdash) ketoacidosis. hyperglycaemic hyperosmolar state and hypoglycaemia. Nat Rev Endocrinol 12, 222-232, 2016.

Virvidakis C, Loukas A, Mayopoulou-Symvoulidou D, Mountokalakis T. Renal responses to bicycle exercise in trained athletes: influence of exercise intensity. Int J Sports Med 7, 86-88, 1986.

Ward KM, Mahan JD, Sherman WM. Aerobic training and diabetic nephropathy in the obese Zucker rat. Ann Clin Lab Sc 24, 266-277, 1994.

Zacharewicz E, Lamon S, Russell AP. MicroRNAs in skeletal muscle and their regulation with exercise, ageing, and disease. Front Physiol 4, 10.3389, 2013.

Xie Y-F, Shu R, Jiang SY, Liu DL, Ni J, Zhang XL. MicroRNA-146 inhibits pro-inflammatory cytokine secretion through IL-1 receptor-associated kinase 1 in human gingival fibroblasts. J Inflamm 10, 20, 2013. 